1984

\title{
Cumulant functions in optical coherence theory
}

CL Mehta

Follow this and additional works at: https://researchrepository.wvu.edu/faculty_publications

\section{Digital Commons Citation}

Mehta, CL, "Cumulant functions in optical coherence theory" (1984). Faculty Scholarship. 110.

https://researchrepository.wvu.edu/faculty_publications/110

This Article is brought to you for free and open access by The Research Repository @ WVU. It has been accepted for inclusion in Faculty Scholarship by an authorized administrator of The Research Repository@WVU. For more information, please contact ian.harmon@mail.wvu.edu. 


\title{
Cumulant functions in optical coherence theory
}

\author{
C L MEHTA* \\ Physics Department, West Virginia University, Morgantown, WV 26506-2063, USA \\ * On leave of absence from the Physics Department, Indian Institute of Technology, New \\ Delhi 110016 , India
}

\begin{abstract}
Cumulant functions are introduced to describe the statistical state of a radiation field. These functions are simply related to the optical coherence functions but have some interesting features. It is shown that if the cumulant functions of all orders greater than some number $N_{0}$ vanish then they also vanish for all orders greater than 2 . Thermal field is the only field having this property. This property holds whether the field is described by a classical stochastic process or by a quantum density operator. Further the particular operator ordering used in defining these cumulant functions for the quantized field affects only the second order cumulant function. To describe the statistical state of a vector field such as partially polarized or unpolarized radiation, one would need to introduce cumulant tensors.
\end{abstract}

Keywords. Cumulant functions; optical coherence; Marcinkiewicz theorem.

\section{Introduction}

In classical description of optical coherence, one considers the radiation field to be governed by a stochastic process. One then introduces the coherence functions (Mandel and Wolf 1965)

$$
G^{(m, n)}\left(x_{1}, \ldots, x_{m} ; y_{1}, \ldots, y_{n}\right)=\left\langle A^{*}\left(x_{1}\right) \ldots A^{*}\left(x_{m}\right) A\left(y_{1}\right) \ldots A\left(y_{n}\right)\right\rangle,
$$

where \langle\rangle$_{e}$ denotes the stochastic ensemble average. The field variable $A(x)$ is the appropriate analytic signal (Gabor 1946) representing the field at the space-time point $x$. For simplicity we consider first the scalar fields such as that of a completely polarized radiation. Generalization to vector fields is straightforward.

In quantum description of the optical coherence, on the other hand, one specifies the statistical state of the radiation field by a density operator $\hat{\rho}$. The coherence function $G^{(m, n)}$ of order $m+n$ is then defined as (cf. Glauber 1963)

$$
\begin{aligned}
G^{(m, n)} & \left(x_{1}, \ldots, x_{m} ; y_{1}, \ldots, y_{n}\right) \\
& =\left\langle\hat{A}^{(-)}\left(x_{1}\right) \ldots \hat{A}^{(-)}\left(x_{m}\right) \hat{A}^{(+)}\left(y_{1}\right) \ldots \hat{A}^{(+)}\left(y_{n}\right)\right\rangle,
\end{aligned}
$$

where $\hat{A}^{(+)}(x)$ and $\hat{A}^{(-)}(x)$ are the positive and negative frequency parts of the field operator $^{\dagger} \hat{A}(x)$ at the space time-point $x$. The angular brackets \langle\rangle$_{q}$ denote the quantum statistical average

$$
\langle\hat{\mathcal{O}}\rangle=\operatorname{tr}(\hat{\rho} \hat{\mathcal{O}})
$$

+ In this paper we denote all operators by a circumflex so as to distinguish them from $c$-number variables. 
Making use of the diagonal coherent state representation of the given density operator (Sudarshan 1963; Mehta 1967), it is possible to rewrite (2) so that the quantum expectation on the right side is expressed as a statistical average over a $c$-number weight function. We may therefore consider the coherence functions $G^{(m, n)}$ in either the classical or the quantum description as essentially the moments of the field variable. In quantum description, however, the operators $\hat{A}^{(+)}(x)$ and $\hat{A}^{(-)}(y)$ in general do not commute (Schweber 1961), and therefore the order in which these operators are arranged while defining the moments is important. In definition (2) above, we have used the normal ordering, i.e. the ordering in which the creation operators stand to the left of the annihilation operators*. If, on the other hand, a different ordering is used in defining the coherence functions, one may use an appropriate $c$-number weight function for such an ordering (Mehta 1968; Agarwal and Wolf 1970). The quantum coherence functions are then the correspondingly ordered moments of the field operator.

Most optical sources emit radiation through thermal excitations, in which case the underlying stochastic process is Gaussian. It is well known that in such cases the second order coherence function $G^{(1,1)}$ determines all the higher order ones (Mehta 1970). This is not so in general. For example when the light originates in stimulated emission, such as from a laser, the underlying stochastic process is not Gaussian. Thus in general the complete statistical properties of the radiation field can only be specified by the coherence functions $G^{(m, n)}$ of all orders.

Related to coherence functions one may also consider quantities which are analogous to the cumulants or the semi-invariants well known in probability theory ( $c f$. Rice 1945). Besides the fact that these cumulant functions determine the statistical properties of the field as much as the coherence functions do, it is found that they have some other interesting features. In particular, we show that except for the second-order cumulant function, these functions do not depend on the ordering in most cases: The normally ordered, antinormally ordered, or the Weyl ordered cumulant functions of order other than 2 are all identical. In $\$ 2$, we introduce the cumulant functions and obtain the relation between these and the coherence functions. It is also shown that the particular operator ordering used in defining the cumulant generating functional affects only the second order cumulant function. In $\S 3$, we show that the only field for which cumulant functions beyond a certain order all vanish is a thermal field for which the underlying stochastic process is Gaussian. Finally in $\$ 4$, we consider the generalization to vector fields, thereby introducing cumulant tensors.

\section{Optical cumulant functions}

Analogous to the characteristic function (Davenport and Root 1958; Lukacs 1960) in probability theory, we introduce the normally ordered characteristic functional

$$
C_{N}(\{\lambda\})=\left\langle\exp \left[\int \lambda(x) \hat{A}^{(-)}(x) \mathrm{d} x\right] \exp \left[-\int \lambda^{*}(x) \hat{A}^{(+)}(x) \mathrm{d} x\right]\right\rangle,
$$

where $x$ denotes the space-time point $\mathbf{r}, t$. The cumulant generating functional $\mathscr{K}_{N}(\{\lambda\})$

\footnotetext{
* It may be noted that the negative frequency part of the field operator contains creation operators while the positive frequency part contains annihilation operators.
} 
is defined as

$$
\mathscr{K}_{N}(\{\lambda\})=\log C(\{\lambda\})
$$

The characteristic functional $C_{N}(\{\lambda\})$ can be expanded in terms of the coherence functions [equation (2)]

$$
\begin{array}{r}
C_{N}(\{\lambda\})=\sum_{m, n=0}^{\infty} \frac{(-1)^{n}}{m ! n !} \int \ldots \int G^{(m, n)}\left(x_{1}, \ldots, x_{m} ; y_{1}, \ldots, y_{n}\right) \\
\lambda\left(x_{1}\right) \ldots \lambda\left(x_{m}\right) \lambda^{*}\left(y_{1}\right) \ldots \lambda^{*}\left(y_{n}\right) \mathrm{d} x_{1} \ldots \mathrm{d} y_{n},
\end{array}
$$

where it is understood that $G^{(0,0)}=1$. We define the cumulant functions $K^{(m, n)}$ as the coefficients in the analogous expansion of the cumulant generating functional

$$
\begin{array}{r}
\mathscr{K}_{N}(\{\lambda\})=\sum_{m, n=0}^{\infty} \frac{(-1)^{n}}{m ! n !} \int \ldots \int K^{(m, n)}\left(x_{1}, \ldots, x_{m} ; y_{1}, \ldots, y_{n}\right) \\
\lambda\left(x_{1}\right) \ldots \lambda\left(x_{m}\right) \lambda^{*}\left(y_{1}\right) \ldots \lambda^{*}\left(y_{n}\right) \mathrm{d} x_{1} \ldots \mathrm{d} y_{n},
\end{array}
$$

where it is understood that $K^{(0,0)}=0$. The coherence function $G^{(m, n)}$ may be obtained from the characteristic functional by taking functional derivatives:

$$
G^{(m, n)}\left(x_{1}, \ldots, x_{m} ; y_{1}, \ldots, y_{n}\right)=\left[\frac{(-1)^{n} \delta^{m+n}}{\delta \lambda\left(x_{1}\right) \ldots \delta \lambda^{*}\left(y_{n}\right)} C_{N}(\{\lambda\})\right]_{\{\lambda\}=0} .
$$

The cumulant functions are also obtained in a similar way

$$
K^{(m, n)}\left(x_{1}, \ldots, x_{m} ; y_{1}, \ldots, y_{n}\right)=\left[\frac{(-1)^{n} \delta^{m+n}}{\delta \lambda\left(x_{1}\right) \ldots \delta \lambda^{*}\left(y_{n}\right)} \mathscr{K}_{N}(\{\lambda\})\right]_{\{\lambda\}=0} .
$$

Making use of the relation (5) we may obtain relations between the coherence functions and the cumulant functions. For example one may readily verify that

$$
\begin{aligned}
& G^{(1,0)}(x)=K^{(1,0)}(x), \\
& G^{(1,1)}(x ; y)=K^{(1,1)}(x ; y)+K^{(1,0)}(x) K^{(0,1)}(y),
\end{aligned}
$$

and in general

$$
\begin{aligned}
& G^{(m, n)}\left(x_{1}, \ldots, x_{m} ; y_{1}, \ldots, y_{n}\right) \\
& \quad=\sum K^{\left(j_{1}, k_{1}\right)}\left(x_{\left(j_{1}\right)} ; y_{\left(k_{1}\right)}\right) \ldots K^{\left(j_{r}, k_{r}\right)}\left(x_{\left(j_{r}\right)} ; y_{\left(k_{r}\right)}\right),
\end{aligned}
$$

where $j_{1}+\ldots+j_{r}=m, k_{1}+\ldots+k_{r}=n, x_{(j)}$ denotes the set of $j$ points $x_{1}, \ldots, x_{j}$ and $y_{(k)}$ denotes the set of $k$ points $y_{1}, \ldots, y_{k}$. The sum on the right side includes all possible different partitions of the space-time points $x_{(m)}, y_{(n)}$. These relations may also be inverted and one could express the cumulant functions in terms of coherence functions. Thus for example one finds that

$$
\begin{aligned}
& K^{(1,0)}(x)=G^{(1,0)}(x) \\
& K^{(1,1)}(x ; y)=G^{(1,1)}(x ; y)-G^{(1,0)}(x) G^{(0,1)}(y) \\
& K^{(2,0)}\left(x_{1}, x_{2}\right)=G^{(2,0)}\left(x_{1}, x_{2}\right)-G^{(1,0)}\left(x_{1}\right) G^{(1,0)}\left(x_{2}\right)
\end{aligned}
$$




$$
\begin{aligned}
K^{(2,1)}\left(x_{1}, x_{2} ; y\right)= & G^{(2,1)}\left(x_{1}, x_{2} ; y\right)-G^{(1,0)}\left(x_{1}\right) G^{(1,1)}\left(x_{1} ; y\right) \\
& -G^{(1,0)}\left(x_{2}\right) G^{(1,1)}\left(x_{1} ; y\right)-G^{(2,0)}\left(x_{1}, x_{2}\right) G^{(0,1)}(y) \\
& +2 G^{(1,0)}\left(x_{1}\right) G^{(1,0)}\left(x_{2}\right) G^{(0,1)}(y)
\end{aligned}
$$

etc. As a direct consequence of the definition of these cumulant functions we find that these functions satisfy the hermiticity property

$$
K^{(m, n)}\left(x_{1}, \ldots, x_{m} ; y_{1}, \ldots, y_{n}\right)=\left[K^{(n, m)}\left(y_{1}, \ldots, y_{n} ; x_{1}, \ldots, x_{m}\right)\right]^{*} .
$$

Further since the coherence functions satisfy wave equations in each of the space time variable $x_{1}, \ldots, x_{m}$ or $y_{1}, \ldots, y_{n}$, the cumulant functions also satisfy similar wave equations.

In place of the normal ordered characteristic functional, one may also consider other orderings such as Weyl or antinormal ordering etc. (Mehta 1968; Agarwal and Wolf 1970). We thus define the characteristic functional

$$
\begin{aligned}
& C_{W}(\{\lambda\})=\left\langle\exp \left\{\int\left[\lambda(x) \hat{A}^{(-)}(x)-\lambda^{*}(x) \hat{A}^{(+)}(x)\right] \mathrm{d} x\right\}\right\rangle, \\
& C_{A}(\{\lambda\})=\left\langle\left\{\exp \left[-\int \lambda^{*}(x) \hat{A}^{(+)}(x) \mathrm{d} x\right]\right\}\left\{\exp \left[\int \lambda(x) \hat{A}^{(-)}(x) \mathrm{d} x\right\}\right]\right\rangle,
\end{aligned}
$$

and the corresponding cumulant generating functionals

$$
\begin{aligned}
& \mathscr{K}_{W}(\{\lambda\})=\log C_{W}(\{\lambda\}), \\
& \mathscr{K}_{A}(\{\lambda\})=\log C_{A}(\{\lambda\}) .
\end{aligned}
$$

The Baker-Housdorff identity, which holds for any two operators $\hat{A}$ and $\hat{B}$ which commute with their commutator, reads (Messiah 1961)

$$
e^{\hat{A}} e^{\hat{B}}=\exp \left\{\hat{A}+\hat{B}+\frac{1}{2}[\hat{A}, \hat{B}]\right\} \text {. }
$$

From the fact that the cumutator $\left[\hat{A}^{(-)}(x), \hat{A}^{(+)}(y)\right]$ is a $c$-number, (Schweber 1961), it therefore follows that

$$
\begin{aligned}
\exp \left\{\int \lambda(x) \hat{A}^{(-)}(x) \mathrm{d} x\right\} \exp \left\{-\int \lambda^{*}(x) \hat{A}^{(+)}(x) \mathrm{d} x\right\} \\
=\exp \left\{\int\left[\lambda(x) \hat{A}^{(-)}(x)-\lambda^{*}(x) \hat{A}^{(+)}(x)\right] \mathrm{d} x\right\} \\
\times \exp \left\{-\frac{1}{2} \iint \lambda(x) \lambda^{*}(y)\left[\hat{A}^{(-)}(x), \hat{A}^{(+)}(y)\right] \mathrm{d} x \mathrm{~d} y,\right. \\
=\exp \left\{-\int \lambda^{*}(x) \hat{A}^{(+)}(x) \mathrm{d} x\right\} \exp \left\{\int \lambda(x) \hat{A}^{(-)}(x) \mathrm{d} x\right\} \\
\times \exp \left\{-\iint \lambda(x) \lambda^{*}(y)\left[\hat{A}^{(-)}(x), \hat{A}^{(+)}(y)\right] \mathrm{d} x \mathrm{~d} y\right\}
\end{aligned}
$$

Hence from (15) and (16) we find that

$$
\begin{aligned}
\mathscr{K}_{N}(\{\lambda\}) & =\mathscr{K}_{W}(\{\lambda\})-\frac{1}{2} \iint \lambda(x) \lambda^{*}(y)\left[\hat{A}^{(-)}(x), \hat{A}^{(+)}(y)\right] \mathrm{d} x \mathrm{~d} y, \\
& =\mathscr{H}_{A}(\{\lambda\})-\iint \lambda(x) \lambda^{*}(y)\left[\hat{A}^{(-)}(x), \hat{A}^{(+)}(y)\right] \mathrm{d} x \mathrm{~d} y .
\end{aligned}
$$

On expansion of (20) and (21) we find that the normal ordered, Weyl ordered and the antinormal ordered cumulant functions are all identical except for $K^{(1,1)}$, where we find that

$$
\begin{aligned}
K_{N}^{(1,1)}(x ; y) & =K_{W}^{(1,1)}(x ; y)+(1 / 2)\left[\hat{A}^{(-)}(x), \hat{A}^{(+)}(y)\right] \\
& =K_{A}^{(1,1)}(x ; y)+\left[\hat{A}^{(-)}(x), A^{(+)}(y)\right] .
\end{aligned}
$$




\section{Marcinkiewicz theorem}

In this section we prove the following theorem: If the cumulant functions $K^{(m, n)}$ vanish identically for all $m$ and $n$ for which $m+n>N$, then they also vanish for all $m$ and $n$ for which $m+n>2$.

This result is a consequence of the positive definiteness of the density operator $\hat{\rho}$ and follows from an application of Marcinkiewicz theorem.

Proof: Since the cumulant functions $K^{(m, n)}$ of order $m+n>2$ are identical whether we consider the normal, Weyl or the antinormal ordering in our definition (see $\$ 2$ above), it suffices to restrict our discussion to antinormally ordered functions. The antinormal ordered characteristic functional is given by

$$
C_{A}(\{\lambda\})=\operatorname{tr}\left[\hat{\rho} \exp \left\{-\int \lambda^{*}(x) \hat{A}^{(+)}(x) \mathrm{d} x\right\} \exp \left\{\int \lambda(x) \hat{A}^{(-)}(x) \mathrm{d} x\right\}\right] .
$$

It is possible to rewrite (24) in the form

$$
C_{A}(\{\lambda\})=\int \phi_{A}(\{v\}) \exp \left\{-\int \lambda^{*}(x) V(x) \mathrm{d} x\right\} \exp \left\{\int \lambda(x) V^{*}(x) \mathrm{d} x\right\} \mathrm{d}^{2}\{v\},
$$

where $\phi_{A}(\{v\})$ is the weight function (Mehta and Sudarshan 1965; Kano 1965)

$$
\phi_{A}(\{v\})=\frac{1}{\pi^{N}}\langle\{v\}|\hat{\rho}|\{v\}\rangle .
$$

Here $|\{v\}\rangle$ is the coherent state, $N$ is the number of active modes (degrees of freedom) and $V(x)$ is the eigenvalue of the operator $\hat{A}^{(+)}(x)$ in the coherent state $|\{v\}\rangle$. Since $\hat{\rho}$ is a positive definite operator, the weight function $\phi_{A}$ is non-negative for all values of $\{v\}$, and hence $C_{A}(\{\lambda\})$ would satisfy all properties of a characteristic functional applicable to classical probability density functions. In particular we make use of the Marcinkiewicz theorem (Marcinkiewicz 1939; Richter 1956; Lukacs 1960) and conclude that the theorem stated in the beginning of this section holds*.

We thus find that if all cumulant functions beyond a certain order vanish identically then the cumulant generating functional can at most be a quadratic. Further if the field is stationary, the cumulant functions of the type $K^{(1,0)}, K^{(0,1)}, K^{(2,0)}, K^{(0,2)}$ also vanish. Hence in such a case the only non-vanishing cumulant function is of the type $K^{(1,1)}$ and the cumulant generating functional is given by

$$
\mathscr{K}(\{\lambda\})=-\iint K^{(1,1)}(x ; y) \lambda(x) \lambda^{*}(y) \mathrm{d} x \mathrm{~d} y .
$$

This is the familiar situation of a thermal field where the underlying stochastic process is Gaussian.

\section{Cumulant tensors}

In the above discussion we have restricted our consideration to scalar fields, i.e. when the field operator $\hat{A}(x)$ is a scalar. This is adequate as long as we are considering

\footnotetext{
* Note that we could not directly apply the Marcinkiewicz theorem in the case of characteristic functional for normal ordering, since the weight function $\phi_{N}(\{v\})$ which is the diagonal coherent state representation of $\hat{\rho}$ is not necessarily positive throughout. Similar is the case for Weyl ordering.
} 
polarized radiation. This consideration may readily be generalized to unpolarized or partially polarized radiation by taking the field operator $\hat{A}(x)$ as a vector. In this case the characteristic functional would be defined as

$$
C_{N}(\{\lambda\})=\left\langle\exp \left[\int \lambda_{i}(x) \hat{A}_{i}^{(-)}(x) \mathrm{d} x\right] \exp \left[-\int \lambda_{i}^{*}(x) \hat{A}_{i}^{(+)}(x) \mathrm{d} x\right]\right\rangle .
$$

When we expand $C_{N}(\{\lambda\})$, we would now include coherence tensors rather than coherence functions

$$
\begin{aligned}
C_{N}(\{\lambda\})=\sum_{m, n=0}^{\infty} \frac{(-1)^{n}}{m ! n !} \int \ldots \int G_{i_{1}, \ldots, i_{m} ; j_{1}, \ldots, j_{n}}^{(m, n)}\left(x_{1}, \ldots, x_{m} ; y_{1}, \ldots, y_{n}\right) \\
\lambda_{i_{1}}\left(x_{1}\right) \ldots \lambda_{i_{m}}\left(x_{m}\right) \lambda_{j_{1}}^{*}\left(y_{1}\right) \ldots \lambda_{j_{n}}^{*}\left(y_{n}\right) \mathrm{d} x_{1} \ldots \mathrm{d} y_{n} .
\end{aligned}
$$

Analogously, while expanding the cumulant generating functional

$$
\begin{aligned}
\mathscr{K}_{N}(\{\lambda\})=\log C_{N}(\{\lambda\})= & \sum_{m, n=0}^{\infty} \frac{(-1)^{n}}{m ! n !} \int \ldots \int K_{i_{1}, \ldots, i_{m} ; j_{1}, \ldots, j_{n}}^{(m, n)}\left(x_{1}, \ldots, x_{m} ; y_{1}, \ldots, y_{n}\right) \\
& \lambda_{i_{1}}\left(x_{1}\right) \ldots \lambda_{i_{m}}\left(x_{m}\right) \lambda_{j_{1}}^{*}\left(y_{1}\right) \ldots \lambda_{j_{n}}^{*}\left(y_{n}\right) \mathrm{d} x_{1} \ldots \mathrm{d} y_{n}
\end{aligned}
$$

we obtain the cumulant tensors. Relations analogous to (10), (11) and (12) may readily be written down for cumulant tensors.

\section{References}

Agarwal G S and Wolf E 1970 Phys. Rev. D2 2161

Davenport W B and Root W L 1958 An introduction to the theory of random signals and noise (New York: McGraw Hill)

Gabor D 1946 J. Inst. Elec. Engrs. 93429

Glauber R J 1963 Phys. Rev. 1312766

Kano Y 1965 J. Math. Phys. 61913

Lukacs E 1960 Characteristic function (London: C. Griffin).

Mandel L and Wolf E 1965 Rev. Mod. Phys. 37231

Marcinkiewicz. J 1939 Math. Z. 44612

Mehta C L 1967 Phys. Rev. Lett. 18752

Mehta C L 1968 J. Phys. A1 385

Mehta C L 1970 in Progress in optics (ed.) E Wolf (Amsterdam: North Holland) Vol. 8, p. 431

Mehta C L and Sudarshan E C G 1965 Phys. Rev. 138 B274

Messiah A 1961 Quantum mechanics (Amsterdam: North Holland) Vol. 1, p. 442

Rice S O 1945 Bell Syst. Tech. J. 23 282; 2446

Rice S O 1945 Bell Syst. Tech. J. 2441

Richter H 1956 Wahrscheinlichkeilstheorie (Berlin: Springer Verlag)

Schweber S S 1961 An introduction to relativistic quantum field theory (New York: Harper and Row)

Sudarshan E C G 1963 Phys. Rev. Lett. 10277 